\title{
Efeito de diferentes temperaturas na germinação de sementes e no crescimento inicial de plântulas de Miconia albicans (Melastomataceae)
}

OLIVEIRA, A.K.M.'*; MOTA, C.M.G.2; AGNES, D.C.2

'Universidade Anhanguera-Uniderp, Programa de Pós-Graduação em Meio Ambiente e Desenvolvimento Regional, Rua Alexandre Herculano, 1400 - Jardim Veraneio, CEP 79037-280, Campo Grande, Mato Grosso do Sul. 'akmorbeckoliveira@gmail.com; 2Universidade Anhanguera-Uniderp, Curso de Ciências Biológicas, Rua Alexandre Herculano, 1400 - Jardim Veraneio, CEP 79037-280, Campo Grande, Mato Grosso do Sul.

\begin{abstract}
RESUMO: Miconia albicans (Sw.) Triana é uma espécie nativa do Brasil utilizada na medicina tradicional em comunidades rurais. O objetivo deste estudo foi investigar a temperatura mais adequada para a germinação de sementes e formação de plântulas de $M$. albicans. Para avaliar o efeito da temperatura sobre a germinação da espécie foram utilizadas 1200 sementes postas a germinar sob temperaturas constantes de $20,25,30$ e $35^{\circ} \mathrm{C}$ e nas temperaturas alternadas de $20-30$ e $25-35^{\circ} \mathrm{C}$. Foram avaliados a percentagem de germinação (PG), o índice de velocidade de germinação (IVG), o tempo médio de germinação (TMG), e o comprimento das raízes primárias e do caule das plântulas. Os resultados encontrados indicaram PG acima de $63 \%$ paras as sementes sob temperaturas de $20,25,30$ e $20-30{ }^{\circ} \mathrm{C}$. Para o IVG, foram observados valores entre 2,17 a 2,93 para as sementes sob temperaturas de 20, 25, 30 e 20-30 ${ }^{\circ} \mathrm{C}$, indicando maior vigor; em relação ao TMG, a maioria dos tratamentos alcançou resultado acima de 15 dias, indicando que as sementes germinaram lentamente. Levando-se em consideração todos os parâmetros avaliados, a temperatura de $25^{\circ} \mathrm{C}$ propiciou o melhor desempenho para as sementes da espécie.
\end{abstract}

Palavras-chave: Cerrado, espécies nativas, plântulas, Melastomataceae, folha-branca.

\begin{abstract}
Effect of different temperatures on seed germination and seedling formation of Miconia albicans (Melastomataceae). The Miconia albicans (Sw.) Triana is a species native to Brazil, which has been used by traditional medicine in rural communities. The aim of this study was to investigate the most suitable temperature for seed germination and seedling development for $M$. albicans. For the evaluation of the effect of temperature on species, a total of 1200 seeds were placed under constant temperatures of $20,25,30$ and $35^{\circ} \mathrm{C}$ and under alternate temperatures of $20-30$ and $25-35^{\circ} \mathrm{C}$. Germination percentage (GP), germination speed index (GSI), average time of germination (ATG) and average length of primary roots and stems of seedlings were evaluated. The results indicate a GP above $63 \%$ for seeds under the constant temperatures of $20,25,30$ and $20-30^{\circ} \mathrm{C}$. For GSI, values between 2.17 and 2.93 were observed for seeds under temperatures of $20,25,30$ and $20-30{ }^{\circ} \mathrm{C}$, which indicates greater vigor; almost all treatments indicated high ATG, longer than 15 days, which demonstrates that the seeds germinated slowly. Taking into consideration all parameters, the temperature of 25 ${ }^{\circ} \mathrm{C}$ provided the best performance for the seeds of this species.
\end{abstract}

Keywords: Cerrado, native species, seedlings, Melastomataceae, Miconia albicans.

\section{INTRODUÇÃO}

Atualmente, a região dos Cerrados é um grande pólo de reprodutibilidade de espécies devido ao desenvolvimento de pesquisas e tecnologias que viabilizaram a utilização dos seus recursos naturais. Neste bioma são reconhecidos mais de 40 tipos fisionômicos de paisagens, com vegetação pouco estudada e apresentando grande potencial medicinal, entre outros usos (Avidos \& Ferreira, 2003; Sano et al., 2008). O interesse econômico pelas espécies vegetais intensificou o extrativismo predatório, levando à extinção ou ameaça de extinção de várias espécies. Porém, nas últimas 
décadas tem aumentado os estudos relacionados à preservação que objetivam domesticar as espécies nativas (Avidos \& Ferreira, 2003), tornando-se necessário o conhecimento das condições de germinação e crescimento inicial para a produção de mudas e sua correta utilização.

Entre as famílias de plantas ocorrentes no bioma, a Melastomataceae Juss. possui cerca de 200 gêneros e 5.000 espécies, sendo Miconia Ruiz

\& Pav. um dos maiores gêneros desta família em número de espécies no Brasil (Souza \& Lorenzi, 2005). Espécies deste gênero ocorrem comumente em áreas de vegetação secundária em bordas ou clareiras naturais e $M$. albicans (Sw.) Triana, conhecida popularmente como folha-branca, é uma das mais comuns, sendo uma espécie arbustiva com 1 a $4 \mathrm{~m}$ de altura (Ellison et al., 1993; Souza \& Lorenzi, 2005) e sementes que possuem cotilédones foliáceos, praticamente sem reservas, uma característica das plantas da família Melastomataceae (Carreira \& Zaidan, 2007).

A espécie $M$. albicans é largamente utilizada na medicina popular contra perturbações digestivas (Siqueira, 1988; Pott \& Pott, 1994). Segundo Vasconcelos et al. (2006), os componentes químicos da espécie possuem atividade analgésica e anti-inflamatória, além de extratos metanólicos de folhas serem altamente reativos contra a bactéria Pseudomonas aeruginosa, além de causar inibição significante contra várias linhagens, incluindo Escherichia coli (Alves et al., 2000).

Outra espécie do mesmo gênero, $M$. fallax DC., também apresenta compostos com atividade analgésica (Andrade e Silva et al., 2002) e, segundo Cunha et al. (2003), os triterpenos extraídos de diferentes espécies do gênero possuem atividade contra tripanossomatídeos.

Uma maneira de contribuir para a conservação da biodiversidade das espécies vegetais está relacionada aos estudos de germinação e crescimento das plântulas envolvendo os principais fatores ambientais que influenciam este processo, como a temperatura. As sementes germinam sob uma temperatura ótima e temperaturas inferiores ou superiores a ela tendem a reduzir a velocidade do processo germinativo, expondo as sementes e plântulas a fatores adversos, o que pode levar à redução no total de germinação e crescimento (Carvalho \& Nakagawa, 2000). A temperatura também é fundamental para o desenvolvimento das estruturas iniciais da plântula, como a raiz primária, e tem um papel crucial no processo de estabelecimento da plântula.

Levando-se em consideração a pequena quantidade de informação referente aos requisitos básicos de $M$. albicans para seu crescimento inicial em áreas do Centro-Oeste do Brasil, o objetivo do presente trabalho foi investigar a temperatura mais adequada para a germinação e crescimento inicial das plântulas desta espécie, visando à produção de mudas mais vigorosas.

\section{MATERIAL E MÉTODOS}

A coleta dos frutos de $M$. albicans foi realizada em setembro de 2010 em uma área de Cerrado na Área de Proteção Ambiental do Guariroba, Campo Grande, Mato Grosso do Sul. Os frutos maduros foram coletados de matrizes e transportados em sacos de papel para o Laboratório de Pesquisa em Sistemas Ambientais e Biodiversidade da Universidade AnhangueraUniderp, em Campo Grande.

Em laboratório, as sementes foram retiradas manualmente dos frutos e foram determinados o grau de umidade das sementes, segundo método de estufa a $105^{\circ} \mathrm{C}$ (Brasil, 2009). As sementes utilizadas para o teste de germinação foram submetidas à assepsia superficial por imersão em hipoclorito de sódio a $2 \%$, por 3 minutos, sendo, em seguida, lavadas em água corrente por 1 minuto. Para a avaliação do efeito da temperatura sobre a germinação, as sementes foram colocadas em câmaras de germinação tipo B.O.D. programadas com as temperaturas constantes de $20,25,30$ e $35^{\circ} \mathrm{C}$, e com as temperaturas alternadas de 20-30 e $25-35{ }^{\circ} \mathrm{C}$. Foram feitas quatro repetições de 50 sementes para os seis tratamentos de temperatura utilizados. Em todos eles o fotoperíodo foi de 12 horas de luz branca (quatro lâmpadas fluorescentes de $20 \mathrm{~W}$ ), sendo que o tratamento de temperatura alternada, 20 e $25^{\circ} \mathrm{C}$, ocorreu no período de escuro. As sementes foram acondicionadas em caixas plásticas transparentes $(11 \times 11 \times 3,5 \mathrm{~cm})$, sobre duas folhas de papel germitest previamente umedecidos com uma solução do fungicida Rovral a $0,1 \% \mathrm{~m} / \mathrm{v}$.

A avaliação da ocorrência de germinação foi diária, com início no primeiro dia após a instalação dos tratamentos e encerrada no $23^{\circ}$ dia. Foram consideradas germinadas as sementes com raiz primária maior ou igual a $2 \mathrm{~mm}$ de comprimento. Para a avaliação do efeito da temperatura sobre o crescimento inicial das plântulas, foram medidos os comprimentos das raízes primárias e do caule dos indivíduos formados, ao fim do experimento de germinação; para isso, foi utilizado um paquímetro digital e medidas 15 plântulas por placa, nos seis tratamentos de temperatura, sendo o resultado expresso em médias das medições.

Foram também avaliados a percentagem de germinação (PG), o índice de velocidade de germinação (IVG) e o tempo médio de germinação

Rev. Bras. PI. Med., Campinas, v.16, n.3, supl. I, p.755-759, 2014. 
(TMG). Não ocorreu necessidade de transformações dos dados de germinação, IVG e TMG, de acordo com os testes de normalidade e homogeneidade de variâncias. Os resultados obtidos foram submetidos à análise de variância (ANOVA) com a utilização do programa estatístico Bioestat 4.0 (Ayres et al., 2004); quando ocorreu significância, foi realizado o teste de Tukey, a 5\% de probabilidade.

\section{RESULTADOS E DISCUSSÃO}

As sementes apresentaram $12 \%$ de umidade, percentual frequentemente encontrado para as sementes segundo Bewley \& Black (1994), entre 5 e $20 \%$. Esse resultado indica que provavelmente não houve influência negativa do fator umidade na germinação.

A percentagem de germinação encontrada para as temperaturas de $20,25,30$ e 20-30 ${ }^{\circ} \mathrm{C}$ foi superior a $63 \%$; já na temperatura de 35 ${ }^{\circ} \mathrm{C}$, nenhuma semente germinou (Tabela 1), demonstrando que esta temperatura está acima da máxima para a espécie, causando danos e/ou morte do embrião. A percentagem de germinação obtida para o tratamento com temperatura alternada de $25-35{ }^{\circ} \mathrm{C}$ foi de $52 \%$, indicando que também houve prejuízo no processo de germinação, mesmo quando a semente foi submetida por um menor período de tempo a temperatura de $35^{\circ} \mathrm{C}$. Segundo Brancalion et al. (2010), as espécies do bioma Cerrado possuem uma maior percentagem de germinação na temperatura em torno de $25^{\circ} \mathrm{C}$, que é considerada ótima para a maior parte das espécies. Assim, os resultados encontrados para $M$. albicans estão de acordo com o esperado.

Os IVG's obtidos nas temperaturas de $20,25,30$ e $20-30^{\circ} \mathrm{C}$ estiveram entre 2,17 a 2,93, indicando maior vigor para a germinação; já a temperatura alternada de $25-35{ }^{\circ} \mathrm{C}$ apresentou menor vigor, igual a 1,5, demonstrando novamente a inadequação de $35^{\circ} \mathrm{C}$ para a germinação das sementes, o que é confirmado pelo valor zero obtido para o IVG sob temperatura igual a $35^{\circ} \mathrm{C}$.
Apesar de $\boldsymbol{M}$. albicans ser encontrada em regiões com temperaturas que ultrapassam os $35^{\circ} \mathrm{C}$, as sementes somente germinam e formam plântulas quando a temperatura ambiente está abaixo deste valor, o que ocorre no período de inverno, entre os meses de junho e setembro, época em que a coleta das sementes foi realizada.

O TMG foi alto para todos os tratamentos, acima de 15 dias (Tabela 1), o que significa que as sementes germinaram lentamente, apesar das diferentes temperaturas testadas. Segundo Carvalho \& Nakagawa (2000), a temperatura ótima para a percentagem de germinação é diferente da temperatura ótima para a velocidade de germinação, sendo mais elevada para esta última, o que não foi observado para $\boldsymbol{M}$. albicans.

Os valores de TMG encontrados podem estar relacionados ao processo de dispersão de frutos pela espécie, que são consumidos por diferentes espécies de aves e roedores (Magnusson \& Sanaiotti, 1987; Goldenberg \& Shepherd, 1998); desta maneira, o processo de ingestão dos frutos pode levar a escarificação química das sementes, acelerando o processo de germinação após as sementes serem defecadas. Este comportamento foi observado em trabalhos de Bocchese et al. (2007), ao avaliarem infrutescências de Cecropia pachystachya Trécul ingeridas por Artibeus lituratus (Olfers, 1818) e Oliveira \& Lemes (2010) com Artibeus planirostris (Spix, 1823) e infrutescências de $\boldsymbol{C}$. pachystachya e Ficus gomelleira Kth. et Bouché, onde registraram diferenças significativas nas taxas e velocidades de germinação, indicando que a ingestão das sementes aumenta a porcentagem e/ou velocidade da germinação. Em pesquisas conduzidas com marsupiais, Cáceres (2002), ao avaliar 11 diferentes tipos de sementes ingeridas por Didelphis albiventris Lund, 1840, também verificou taxas de germinação superiores a $67 \%$ para seis espécies. Resultados similares foram apresentados por Oliveira e Leme (2013), ao estudarem $\boldsymbol{D}$. albiventris e sementes consumidas de Rapanea ferruginea

TABELA 1. Valores médios de percentagem de germinação (PG), índice de velocidade de germinação (IVG), tempo médio de germinação em dias (TMG) e comprimento da raiz primária e do caule de sementes de Miconia albicans sob seis regimes de temperatura

\begin{tabular}{cccccc}
\hline Temperatura $\left({ }^{\circ} \mathrm{C}\right)$ & PG $(\%)$ & IVG & TMG (dias) & $\begin{array}{c}\text { Comprimento da } \\
\text { raiz primária }(\mathrm{cm})\end{array}$ & $\begin{array}{c}\text { Comprimento do } \\
\text { caule }(\mathrm{cm})\end{array}$ \\
\hline 20 & $69 \mathrm{ab}$ & $2,2 \mathrm{a}$ & $17 \mathrm{a}$ & $1,0 \mathrm{~b}$ & $0,7 \mathrm{ab}$ \\
25 & $76 \mathrm{a}$ & $2,9 \mathrm{a}$ & $15 \mathrm{a}$ & $1,5 \mathrm{a}$ & $0,8 \mathrm{a}$ \\
30 & $65 \mathrm{~b}$ & $2,3 \mathrm{a}$ & $16 \mathrm{a}$ & $0,6 \mathrm{c}$ & $0,8 \mathrm{a}$ \\
35 & $0,0 \mathrm{~d}$ & $0,0 \mathrm{c}$ & $0,0 \mathrm{~b}$ & $0,0 \mathrm{e}$ & $0,0 \mathrm{c}$ \\
$20-30$ & $64 \mathrm{~b}$ & $2,2 \mathrm{a}$ & $15 \mathrm{a}$ & $0,8 \mathrm{bc}$ & $0,7 \mathrm{ab}$ \\
$25-35$ & $52 \mathrm{c}$ & $1,5 \mathrm{~b}$ & $17 \mathrm{a}$ & $0,2 \mathrm{~d}$ & $0,5 \mathrm{~b}$ \\
\hline
\end{tabular}

Médias seguidas pela mesma letra na coluna não diferem estatisticamente entre si (Tukey, $p>0,05)$.

Rev. Bras. PI. Med., Campinas, v.16, n.3, supl. I, p.755-759, 2014. 


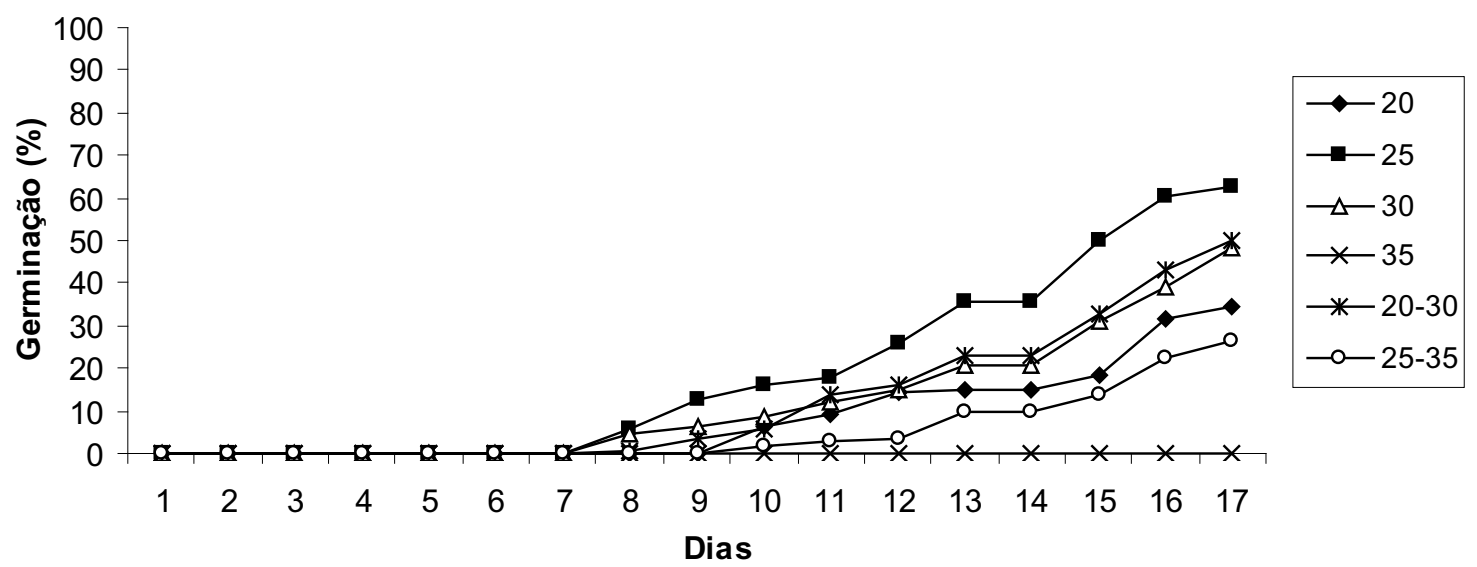

FIGURA 1. Percentagem acumulada de germinação das sementes de $M$. albicans em seis temperaturas.

(Ruiz et Pav.) (Myrcinaceae), resultando em maiores taxas e velocidades de germinação após passagem pelo sistema digestório.

As sementes iniciaram os processos germinativos quase simultaneamente nas várias temperaturas testadas, sendo no $10^{\circ}$ dia para as temperaturas de 20 e $25-35^{\circ} \mathrm{C}$ e no oitavo dia para as temperaturas de 25,30 e $20-30{ }^{\circ} \mathrm{C}$, com sementes germinando até o $17^{\circ}$ dia (Figura 1). A germinação ocorreu diariamente para quase todas as temperaturas, indicando que a espécie distribui a germinação por vários dias. Segundo Brancalion \& Marcos Filho (2008), a distribuição por um período de tempo maior pode ser mais adequado para uma espécie, pois permite maior chance das plântulas encontrarem um ambiente favorável ao seu estabelecimento.

Em estudo com a mesma espécie, porém coletada na região de Mogi Guaçu-SP, distante 166 km da cidade de São Paulo, sudeste do Brasil, Carreira \& Zaidan (2007) encontraram resultados semelhantes e afirmaram que, com relação à temperatura, a germinação das sementes ocorreu preferencialmente entre 20 e $30^{\circ} \mathrm{C}$, além da alternada $20-30^{\circ} \mathrm{C}$, porém considerando apenas as temperaturas de 25 e $30^{\circ} \mathrm{C}$ de melhor velocidade de germinação, o que não foi observado para as sementes coletadas na região de Campo GrandeMS. Tal resultado indica que, dependendo da região de coleta, a espécie pode apresentar características germinativas distintas, como sendo uma adaptação ao ambiente. Segundo Figliolia et al. (1993) e Carvalho \& Nakagawa (2000), o ambiente de origem das sementes pode ter grande influência sobre seu comportamento e percentuais de germinação, o que foi observado para a espécie em questão.

A avaliação do crescimento da plântula por meio das medidas do comprimento médio da raiz primária e do caule (Tabela 1) demostrou que a temperatura de $25^{\circ} \mathrm{C}$ foi a que apresentou o maior crescimento da raiz primária, atingindo $1,5 \mathrm{~cm}$. Já o crescimento da parte aérea, igual a $8 \mathrm{~cm}$, foi similar nas temperaturas de 20,30 e $20-30^{\circ} \mathrm{C}$, indicando que esta estrutura é menos sensível às diferentes temperaturas. Já na temperatura de $35^{\circ} \mathrm{C}$ pode-se considerar que a atividade respiratória tenha se sobressaído em relação aos demais processos fisiológicos como a fotossíntese e/ou que as sementes tenham quase esgotado ou esgotado suas reservas antes de germinar, resultando em plântulas de menor tamanho. De acordo com Cassaro-Silva (2001), em temperaturas muito elevadas em relação a aquelas que a espécie tolera, a energia contida nas células ou sistemas de membranas celulares da semente fica cada vez menos favorável ao crescimento do embrião, até atingir a temperatura máxima em que toda a energia é dissipada, limitando a germinação.

Levando-se em consideração todos dos parâmetros avaliados, a temperatura de $25^{\circ} \mathrm{C}$ é a melhor para a germinação de sementes e desenvolvimento inicial das plântulas da espécie, produzindo mudas mais desenvolvidas em menor espaço de tempo, o que leva a propor que em iniciativas de propagação da espécie, essa temperatura seja utilizada.

\section{AGRADECIMENTOS}

Os autores agradecem a Universidade Anhanguera-Uniderp pelo financiamento do projeto GIP (Grupo Interdisciplinar de Pesquisa) e ao Conselho Nacional de Desenvolvimento Científico e Tecnológico (CNPq), pela bolsa Produtividade em Pesquisa concedida. 


\section{REFERÊNCIA}

ALVES, T.M.A. et al. Biological screening of brazilian medicinal plants. Memórias do Instituto Oswaldo Cruz, v. 95, n. 3, p.367-373, 2000.

ANDRADE E SILVA, M.L. et al. Evaluation of the analgesic activity of an ethanol extract of Miconia fallax. Bollettino Chimico Farmaceutico, v. 141, n. 2, p. 158-160, 2002.

AVIDOS, M.F.D.; FERREIRA, L.T. Frutos do Cerrado: preservação gera muitos frutos. Biotecnologia, Ciência e Desenvolvimento, 2003. 41p.

AYRES, M. et al. BioEstat 4.0: Aplicações estatísticas nas áreas das ciências biológicas e médicas. Manaus: Sociedade Civil Mamirauá, 2004. 364p.

BEWLEY, J.D.; BLACK, M. Seeds: physiology of development and germination. New York and London: Plenum Press, 1994. 445p.

BOCCHESE, R.A.; OLIVEIRA, A.K.M.; VICENTE, E.C. Taxa e velocidade de germinação de sementes de Cecropia pachystachya Trécul (Cecropiaceae) ingeridas por Artibeus lituratus (Olfers, 1818) (Chiroptera: Phyllostomidae). Acta Scientiarum, Biological Sciences, v. 29, n. 4, p. 395-399, 2007.

BRANCALION, P.H.S.; MARCOS FILHO, J. Distribuição da germinação no tempo: causas e importância para a sobrevivência das plantas em ambientes naturais. Informativo ABRATES, v. 18, n. 1, 2, 3, p. 11-17, 2008.

BRANCALION, P.H.S.; NOVEMBRE, A.D.L.C.; RODRIGUES, R.R. Temperatura ótima de germinação de sementes de espécies arbóreas brasileiras. Revista Brasileira de Sementes, v. 32, n. 4, p. 15-21, 2010.

BRASIL. Ministério da Agricultura, Pecuária e Abastecimento. Regras para análise de sementes. Brasília: Secretaria de Defesa Agropecuária. Mapa/ ACS, 2009. 399p.

CÁCERES, N. C. Food habits and seed dispersal by the white-eared opossum Didelphis albiventris in Southern Brazil. Studies on Neotropical Fauna and Environment, v. 37, n. 2, p. 97-104, 2002.

CARREIRA, R.C.; ZAIDAN, L.B.P. Germinação de sementes de espécies de Melastomataceae de Cerrado sob condições controladas de luz e temperatura. Hoehnea, v. 34, n. 3, p. 261-269, 2007.

CARVALHO, N.M.; NAKAGAWA, J. Sementes: ciência, tecnologia e produção. Jaboticabal: FUNEP, 2000. $588 p$.
CASSARO-SILVA, M. Efeito da temperatura na germinação de sementes de manduirana (Senna macranthera (Collad.) Irwin et Barn. - Caesalpiniaceae). Revista Brasileira de Sementes, v. 23, n. 1, p. 92-99, 2001.

CUNHA, W.R. et al. In vitro trypanocidal activity of triterpenes from Miconia species. Planta Medica, v. 69, n. 5, p. 470-472, 2003.

ELLISON, A.M. et al. Seed and seedling ecology of Neotropical Melastomataceae. Ecology, v. 74, n. 6, p. 1733-1749, 1993.

FIGLIOLIA, M.B.; OLIVEIRA, E.C.; PINÃ-RODRIGUES, F.C.M. Análise de sementes. In: AGUIAR, I.B.; PINÃRODRIGUES, F.C.M.; FIGLIOLIA, M.B. (Coord.). Sementes Florestais Tropicais. Brasília: Associação Brasileira de Tecnologia de Sementes, p. 137-174. 1993.

GOLDENBERG, R.; SHEPHERD, G.J. Studies on the reproductive biology of Melastomataceae in cerrado vegetation. Plant Systematics and Evolution, v. 211, n. 1-2, p. 13-29, 1998.

MAGNUSSON, W.E.; SANAIOTTI, T.M. Dispersal of Miconia seeds by the rat Bolomys lasiurus. Journal of Tropical Ecology, v. 3, n. 3, p. 277-278, 1987.

OLIVEIRA, A.K.M.; LEMES, F.T.F. Artibeus planirostris como dispersor e indutor de germinação em uma área do Pantanal do Negro, Mato Grosso do Sul. Revista Brasileira de Biociências, v. 8, n. 1, p. 49-52, 2010.

OLIVEIRA, A.K.M.; LEMES, F.T.F. Didelphis albiventris como indutor de germinação de Rapanea ferruginea (Myrcinaceae) em área de Cerrado, Mato Grosso do Sul, Brasil. Iheringia, Série Zoologia, Porto Alegre, v. 103, n. 4, p. 361-366, 2013.

POTT, A.; POTT, V.J. Plantas do Pantanal. Corumbá: EMBRAPA/CPAP-SPI, 1994. 320p.

SANO, S.M.; RIBEIRO, J.P.; ALMEIDA, S.P. (Eds.). Cerrado: ecologia e flora. Planaltina: Embrapa Cerrados, v. 1, 2008. 406p.

SIQUEIRA, J.C. Utilização popular das plantas do cerrado. São Paulo: Edusp/ Fapesp, 1988. 194p.

SOUZA, V.C.; LORENZI, H. Botânica sistemática: guia ilustrado para identificação das famílias de Angiospermas da flora brasileira, baseado em APG II. Nova Odessa: Instituto Plantarum, 2005. 640p.

VASCONCELOS, M.A. et al. In vivo analgesic and antiinflammatory activities of ursolic acid and oleanoic acid from Miconia albicans (Melastomataceae). Zeitschrift für Naturforschung - Section C, v. 61, n. 7-8, p. 477482, 2006. 